\title{
Appendix 1. Stamp assemblages
}

T A B L E I . L A N A U T I Q U E

\begin{tabular}{|c|c|c|c|c|c|c|c|c|c|c|c|c|c|}
\hline Potter & Die number & Transcription & $\begin{array}{r}\text { Reference } \\
\text { to Fiches et } \\
\text { al. } 1978 \\
\end{array}$ & $\begin{array}{l}\text { Drag. } \\
24 / 25\end{array}$ & $\begin{array}{r}\text { Drag. } \\
27\end{array}$ & cups & $\begin{array}{l}\text { Drag. } \\
15 / 17\end{array}$ & $\begin{array}{r}\text { Drag. } \\
18\end{array}$ & $\begin{array}{l}\text { Drag. } \\
15 / 17 \\
\text { or } 18 \\
\end{array}$ & $\begin{array}{r}\text { Drag. } \\
29\end{array}$ & $\begin{array}{l}\text { Drag. } \\
2 / 21\end{array}$ & $\begin{array}{r}\text { Drag. } \\
16\end{array}$ & Total \\
\hline Acutus i & Acutus i 30b & ACVI & 1 & & 3 & & & & & & & & 3 \\
\hline \multirow[t]{3}{*}{ Acutillus } & Acutillus 1a & OF ACVTIL & 2 & & & & & & 6 & & & & 6 \\
\hline & Acutillus 2a & OFACVTIL & 3 & & & & & 1 & 1 & & & & 2 \\
\hline & unidentified & ALB & 4 & 2 & 1 & & & & & & & & 3 \\
\hline Albanus ii & $\begin{array}{l}\text { Albanus ii } \\
10 \mathrm{~b}\end{array}$ & ALBANI & 5 & 2 & & & & & & & & & 2 \\
\hline \multirow[t]{3}{*}{ Albinus iii } & Albinus iii 4a & OFALBINI & 6 & & & & & & 9 & & & & 9 \\
\hline & Albinus iii 5a & OFALBIN & 7 & 5 & 10 & & & & & & & & 15 \\
\hline & Albinus iii 4c & OFALBINI & 8 & & & & & & & 1 & & & 1 \\
\hline Ardanus & Ardanus 4a & OARDAN & 9 & 3 & & & 1 & & & & & & 4 \\
\hline \multirow[t]{5}{*}{ Bassus ii } & Bassus ii 13b & BASSIO & 10 & 1 & & & & & & & & & 1 \\
\hline & Bassus ii $7 \mathrm{~b}$ & OFBAS & 11 & 4 & & & & & & & & & 4 \\
\hline & Bassus ii $4 c$ & OFBASSI & 12 & & 1 & & & & & & & & 1 \\
\hline & Bassus ii 4j & OF.BASSI & 13 & & 5 & & & & & & & & 5 \\
\hline & Bassus ii $4 \mathrm{~g}$ & OFBASSI & 14 & & & & & & 5 & & & & 5 \\
\hline Bellicus i & Bellicus i 6a & OFBELIC & 15 & 1 & & & & & & & & & 1 \\
\hline (Bionis) & ? 0.BI & $0 . \mathrm{BI}$ & 16 & 7 & 2 & & & & & & & & 9 \\
\hline Capito i & Capito i 1a & CAPITO.VA & 17 & & & & & & & 1 & & & 1 \\
\hline Carillus ii & Carillus ii 2a & CARLLI & 18 & & & & & & 1 & & & & 1 \\
\hline Chrestus & Chrestus 8a & CHRES & 19 & & 1 & & & & & & & & 1 \\
\hline Cocus i & Cocus i 11a & COCI.OFIC & 20 & & & & & & & 7 & & & 7 \\
\hline Cocis & Cocis 1a & OFCOCIS & 21 & & & & & & & & 1 & & 1 \\
\hline $\begin{array}{l}\text { Cosius Urap- } \\
\text { pus }\end{array}$ & $\begin{array}{l}\text { Cosius Urap- } \\
\text { pus 1a }\end{array}$ & COSIVS.VRAP & 22 & & 9 & 1 & & & & & & & 10 \\
\hline \multirow[t]{3}{*}{ Cotto i } & Cotto i 6-a & сото & 23 & & 6 & & & & & & & & 6 \\
\hline & Cotto i $4 b$ & сотто & 24 & 7 & & & & & & & & & 7 \\
\hline & Cotto i 3-a & СОTTOF & 25 & 6 & 3 & & & & & & & & 9 \\
\hline Crobus & Crobus 1a & CROBI & 26 & 9 & 3 & & & & & & & & 12 \\
\hline Dab(i)us & Dab(i)us 1-a & OFDABI & 27 & & & 1 & & & & & & & 1 \\
\hline \multirow[t]{4}{*}{ Damonus } & Damonus 15l & DAMO & 28 & 2 & & & & & & & & & 2 \\
\hline & $\begin{array}{l}\text { Damonus } \\
14 c\end{array}$ & DAMON & 29 & 5 & & & & & 2 & & & & 7 \\
\hline & $\begin{array}{l}\text { Damonus } \\
13 \mathrm{~m}\end{array}$ & DAMONI & 30 & & 1 & & & & & & & & 1 \\
\hline & $\begin{array}{l}\text { Damonus } \\
11 \mathrm{e}\end{array}$ & DAMONUS & 31 & & & & & & & & & 1 & 1 \\
\hline Darra(ntus) & $\begin{array}{l}\text { Darra(ntus) } \\
\text { 2a }\end{array}$ & DARRAFE & 32 & & & 1 & & & & & & & 1 \\
\hline Dior-2a & Dior- 2a & DIORIF & 33 & & 7 & & & & & & & & 7 \\
\hline
\end{tabular}




\begin{tabular}{|c|c|c|c|c|c|c|c|c|c|c|c|c|c|}
\hline Potter & Die number & Transcription & $\begin{array}{r}\text { Reference } \\
\text { to Fiches et } \\
\text { al. } 1978\end{array}$ & $\begin{array}{l}\text { Drag. } \\
24 / 25\end{array}$ & $\begin{array}{r}\text { Drag. } \\
27\end{array}$ & cups & $\begin{array}{l}\text { Drag. } \\
15 / 17\end{array}$ & $\begin{array}{r}\text { Drag. } \\
18\end{array}$ & $\begin{array}{l}\text { Drag. } \\
15 / 17 \\
\text { or } 18\end{array}$ & $\begin{array}{r}\text { Drag. } \\
29\end{array}$ & $\begin{array}{r}\text { Drag. } \\
2 \mid 21\end{array}$ & $\begin{array}{r}\text { Drag. } \\
16\end{array}$ & Total \\
\hline Esgen- & Esgen- 1a & ESGEN & 34 & 16 & & & & & & & & & 16 \\
\hline Felix i & Felix i 24a & FELICISMA & 35 & & & & & & & 4 & & & 4 \\
\hline \multirow[t]{2}{*}{ Festus i } & Festus i 6a & FESTVS & 36 & & & & & 1 & 1 & & & & 2 \\
\hline & Festus i 4a & FESTVSF & 37 & & 4 & & & & & & & & 4 \\
\hline Firmo i & Firmo i [9-a] & FIRMO & 38 & & & & & & 1 & & & & 1 \\
\hline Gallincanus ii & $\begin{array}{l}\text { Gallicanus } \\
\text { ii } 10 \mathrm{a}\end{array}$ & GALLICANI & 39 & & 6 & & & & & & & & 6 \\
\hline Gallus ii & Gallus ii 6a & GALLI.MAN & 40 & & & & & 3 & & & & & 3 \\
\hline Ingenuus ii & $\begin{array}{l}\text { Ingenuus } \\
\text { ii } 13 \mathrm{~b}\end{array}$ & OF.INGEN & 41 & & & & & & 1 & & & & 1 \\
\hline lucundus ii & $\begin{array}{l}\text { lucundus } \\
\text { ii } 6 c\end{array}$ & IUCUND & 42 & & & & & & 1 & & & & 1 \\
\hline Lartius & Lartius 1a & LARTIM & 43 & & & & & 1 & 2 & & & & 3 \\
\hline \multirow[t]{3}{*}{ Licinus } & Licinus 49b & LICNVS & 44 & & 2 & & & & & & & & 2 \\
\hline & Licinus $25 c$ & OFLICN & 45 & & 1 & & & & & & & & 1 \\
\hline & Licinus 39b & LICINI & 46 & & & 1 & & & & & & & 1 \\
\hline Manertus & Manertus 3a & M()ERTVSF & 47 & & 1 & & & & & & & & 1 \\
\hline \multirow[t]{2}{*}{ Martialis i } & $\begin{array}{l}\text { Martialis i } \\
11 a\end{array}$ & MARTIALISF & 48 & & & & & & 1 & & & & 1 \\
\hline & Martialis i 6a & $\begin{array}{l}\text { MARTIAL- } \\
\text { ISVA }\end{array}$ & 49 & & & & 1 & & & & & & 1 \\
\hline Quartus iii & $\begin{array}{l}\text { Quartus iii } \\
8 a^{\prime}\end{array}$ & ()ARTVSF & 50 & & & & & & 1 & & & & 1 \\
\hline Melain- & Melain-1a & MELAINIMA & 51 & & & & & & & 1 & & & 1 \\
\hline illiterate & illiterate & MO & 52 & & 7 & & & & & & & & 7 \\
\hline \multirow[t]{5}{*}{ Modestus i } & $\begin{array}{l}\text { Modestus } \\
\text { i } 26 \mathrm{~b}\end{array}$ & MODE & 53 & & 5 & & & & & & & & 5 \\
\hline & $\begin{array}{l}\text { Modestus } \\
\text { i } 9 g\end{array}$ & OFMOD & 54 & & 5 & 2 & & & & & & & 7 \\
\hline & $\begin{array}{l}\text { Modestus } \\
\text { i } 2 \mathrm{~d}\end{array}$ & OFMODES() & 55 & & & & & & & 1 & & & 1 \\
\hline & $\begin{array}{l}\text { Modestus } \\
\text { i } 4 \mathrm{e}\end{array}$ & OFMODES & 56 & & & & & & 14 & & & & 14 \\
\hline & $\begin{array}{l}\text { Modestus } \\
\text { i } 4 \mathrm{~d}\end{array}$ & OFMODES & 57 & & & 5 & & & & & & & 5 \\
\hline Paestor & Paestor 2a & PAESTOR & 58 & & 4 & 2 & & & & & & & 6 \\
\hline \multirow[t]{7}{*}{ Primus iii } & Primus iii 46i & PRIM & 59 & 1 & & & & & & & & & 1 \\
\hline & $\begin{array}{l}\text { (Primus iii } \\
46 \text { ) }\end{array}$ & PRIM & 60 & & 16 & & & & & & & & 16 \\
\hline & $\begin{array}{l}\text { Primus iii } \\
12 \mathrm{e}\end{array}$ & OFPRIMI & 61 & & & & & & & 1 & & & 1 \\
\hline & $\begin{array}{l}\text { Primus iii } \\
12 n\end{array}$ & OFPRIMI & 62 & & & & & 1 & & & & & 1 \\
\hline & $\begin{array}{l}\text { Primus iii } \\
120\end{array}$ & OFPRIMI & 63 & & & & & & 1 & & & & 1 \\
\hline & Primus iii 18j & OFPRIM & 64 & & & & & & 1 & & & & 1 \\
\hline & Primus iii 21j & OF.PRM & 65 & 13 & 2 & & & & & & & & 15 \\
\hline Quintus iii & Quintus iii 9a & QVI & 66 & & 12 & & & & & & & & 12 \\
\hline Regenus & Regenus 3-a & REGENI & 67 & & & & & 5 & 10 & & & & 15 \\
\hline Sabinus iii & $\begin{array}{l}\text { Sabinus iii } \\
\text { 21a }\end{array}$ & O.SABI & 68 & & 24 & 2 & & & & & & & 26 \\
\hline $\begin{array}{l}\text { G. Salarius } \\
\text { Aptus }\end{array}$ & $\begin{array}{l}\text { G. Salarius } \\
\text { Aptus } 10 a\end{array}$ & SAL.ARTI & 69 & & & & & 1 & & & & & 1 \\
\hline
\end{tabular}




\begin{tabular}{|c|c|c|c|c|c|c|c|c|c|c|c|c|c|}
\hline Potter & Die number & Transcription & $\begin{array}{r}\text { Reference } \\
\text { to Fiches et } \\
\text { al. } 1978 \\
\end{array}$ & $\begin{array}{l}\text { Drag. } \\
24 / 25\end{array}$ & $\begin{array}{r}\text { Drag. } \\
27\end{array}$ & cups & $\begin{array}{l}\text { Drag. } \\
15 / 17\end{array}$ & $\begin{array}{r}\text { Drag. } \\
18\end{array}$ & $\begin{array}{l}\text { Drag. } \\
15 / 17 \\
\text { or } 18 \\
\end{array}$ & $\begin{array}{r}\text { Drag. } \\
29\end{array}$ & $\begin{array}{l}\text { Drag. } \\
2 / 21\end{array}$ & $\begin{array}{r}\text { Drag. } \\
16\end{array}$ & Total \\
\hline Salvetus i & $\begin{array}{l}\text { Salvetus i } \\
11 \mathrm{~h}\end{array}$ & SALVI & 70 & & & 5 & & & & & & & 5 \\
\hline \multirow[t]{2}{*}{ Scotnus } & Scotnus 4a & SCOTNVS & 71 & & & & & & & 4 & & & 4 \\
\hline & Scotnus 3a & SCOTNI & 72 & & & 2 & 2 & & & & & & 4 \\
\hline \multirow[t]{2}{*}{ Senecio } & Senecio 8b & SENECI & 73 & 3 & & & & & & & & & 3 \\
\hline & Senecio 8a & SENECI & 74 & 21 & & & & & & & & & 21 \\
\hline Senilis i & Senilis i 2b & SENILISF & 75 & & 1 & & & & & & & & 1 \\
\hline \multirow[t]{4}{*}{ Silvanus i } & $\begin{array}{l}\text { Silvanus i } \\
15 b\end{array}$ & SILVAN & 76 & 1 & & & & 1 & & & & & 2 \\
\hline & $\begin{array}{l}\text { Silvanus i } \\
17 \mathrm{~b}\end{array}$ & SILVAN & 77 & 5 & & & & & & & & & 5 \\
\hline & Silvanus i 6b & SILVANI.OF & 78 & & & & & & & 1 & & & 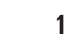 \\
\hline & Silvanus i 3c & OFSILVAN & 79 & & & & 2 & & & & & & 2 \\
\hline Silvinus i & Silvinus i 1a & OFSILVAN & 80 & & & & 2 & & & & & & . \\
\hline Successus ii & $\begin{array}{l}\text { Successus } \\
\text { ii } 7 a\end{array}$ & SVCCES & 81 & & & 1 & & & & & & & 1 \\
\hline (Tertius) & (TERTIUS.FE) & TERTIVS.F & 82 & & & & & 2 & & & & & 2 \\
\hline (Virtus) & (IRTVS) & IRTVS & 83 & & & 3 & & & & & & & 3 \\
\hline illiterate & illiterate & IVIIX & 84 & 7 & 14 & & & & & & & & 21 \\
\hline \multirow[t]{2}{*}{ Labio } & Labio 3a & OF.L.ABIO & 85 & & & & & 1 & & & & & 1 \\
\hline & Labio 3b & OF.LABIO & 86 & & & & & & 1 & & & & 1 \\
\hline \multirow[t]{5}{*}{ unidentified } & unidentified & unidentified & 87 & & 1 & & & & & & & & 1 \\
\hline & unidentified & unidentified & 88 & & 1 & & & & & & & & 1 \\
\hline & unidentified & unidentified & 89 & & 1 & & & & & & & & 1 \\
\hline & unidentified & unidentified & 90 & & 1 & & & & & & & & 1 \\
\hline & & & & 121 & 160 & 26 & 8 & 17 & 59 & 21 & 1 & 1 & 414 \\
\hline
\end{tabular}


T A B L E 2. C A L A C U L I P I V

\begin{tabular}{|c|c|c|c|c|c|c|c|c|c|c|}
\hline Potter & Die number & Transcription & $\begin{array}{r}\text { Reference to } \\
\text { Nieto et al. } \\
1989\end{array}$ & $\begin{array}{l}\text { Drag. } \\
24 / 25\end{array}$ & Drag. 27 & $\begin{array}{l}\text { Drag. } \\
15 / 17\end{array}$ & Drag. 18 & Drag. 29 & $\begin{array}{r}\text { Hermet } \\
1\end{array}$ & Total \\
\hline Albanus ii & Albanus ii 18a & ALBAN & 1.1 & & & & & & 2 & 2 \\
\hline Caius i & (Caius i/Gaius i) & OFCAIV & 31.1 & & & & 1 & & & 1 \\
\hline $\begin{array}{l}\text { (C. Valerius } \\
\text { Albanus) }\end{array}$ & (C.VAL.ALBAN) & C.VAL.ALBAN & 34.1 & & & & & 2 & & 2 \\
\hline Caius i & (Caius i/Gaius i) & CAI() & 5.2 & & & & 1 & & & 1 \\
\hline Calvus i & Calvus i & OFCALVS & 35.1 & & & & & 2 & & 2 \\
\hline Cabucatus ii & Cabucatus ii a & CABVCATI & 5.1 & & & & 15 & & & 15 \\
\hline \multirow[t]{3}{*}{ Logirnus } & Logirnus 3a' & 0 CIRNI & 4.1 & & & 68 & 20 & & & 88 \\
\hline & Logirnus 3a & IO CIRNIO & 4.2 & & & & 17 & & & 17 \\
\hline & Logirnus 5a & $\mathrm{IO} C() \mathrm{NI}$ & 4.3 & & & & 2 & & & 2 \\
\hline Coelus ii & Coelus ii 1a & OFCOELI & 36.1 & & & & & 1 & & 1 \\
\hline cosius Rufinus & $\begin{array}{l}\text { Cosius Rufinus } \\
8 a^{\prime}\end{array}$ & OSFRV & 11.1 & & & & 6 & & & 6 \\
\hline Germanus i & Germanus i $27 c^{\prime}$ & GERMANI & 8.1 & & & & 1 & & & 1 \\
\hline Im- $\mathrm{i}$ & Im-i 1-a & IM & 24.1 & & 1 & & & & & 1 \\
\hline Nivius & (Nivius 1a) & NIV? & 28.1 & & 8 & & & & & 8 \\
\hline \multirow[t]{8}{*}{ lucundus iii } & lucundus iii $5 f$ & OF.IVCVN & 2.1 & & & & 162 & & & 162 \\
\hline & lucundus iii $5 b$ & OF.IVCVN & 2.2 & & & & 267 & & & 267 \\
\hline & lucundus iii $5 b$ & OF.IVCVN & 2.3 & & & 8 & & & & 8 \\
\hline & lucundus iii $5 c$ & OF.IVCVN & 2.4 & & 220 & & & & & 220 \\
\hline & (lucundus iii) & ()IVCVN() & 2.5 & & 8 & & & & & 8 \\
\hline & lucundus iii 3b & OF.IVCVNDI & 2.6 & 193 & 7 & & & & & 200 \\
\hline & lucundus iii 3b & OFIVCVNDI & 2.7 & 19 & 27 & & & & & 46 \\
\hline & lucundus iii 3a & OFIVCVNDI & 2.8 & & & & & 40 & & 40 \\
\hline Mevius (Mevus) & $\begin{array}{l}\text { Mevius (Mevus) } \\
\text { 3a }\end{array}$ & MIIM & 25.1 & & 11 & & & & & 11 \\
\hline Mommo & Mommo 9i & OFMOM & 9.1 & & & & 46 & 2 & & 48 \\
\hline \multirow[t]{2}{*}{ Senicio } & Senicio 6a' & ()ENICI & 15.1 & & 1 & & & & & 1 \\
\hline & Senicio $5 b$ & ()ENICIO & 15.2 & 3 & & & & & & 3 \\
\hline Pass(i)enus & Passienus 5a & OFPASSEN & 38.1 & & & & & 34 & & 34 \\
\hline Patricius i & Patricius i 3d & OF.PATRICI & 13.1 & & & 2 & & & & 2 \\
\hline Peregrinus i & Peregrinus i 3a & PE()RIV & 20.1 & & & & 3 & & & 3 \\
\hline Ponteius & Ponteius 1a & OFPONE()I & 21.1 & & & & 2 & & & 2 \\
\hline Primus iii & Primus iii 12r & OFPRIMI & 39.1 & & & & & 5 & & 5 \\
\hline Primulus i & Primulus i 4b & PRIMVLI & 7.1 & & & & 11 & & & 11 \\
\hline Quintio i & Quintio i 1b & QVINTIO & 40.1 & & & & & 2 & & 2 \\
\hline Crispus iii & Crispus iii 7a' & RISPI.MA & 41.1 & & & & & 2 & & 2 \\
\hline \multirow[t]{2}{*}{ Rufinus iii } & Rufinus iii 2b & OF.RVFIN & 42.1 & & & & & 4 & & 4 \\
\hline & Rufinus iii 3a & OF.RVFNI & 10.1 & & & & 8 & & & 8 \\
\hline Sabinus iii & Sabinus iii 8b & OF.SABINI & 12.1 & & & 7 & & & & 7 \\
\hline \multirow[t]{2}{*}{ Secundus ii } & $\begin{array}{l}\text { Secundus ii } \\
11 a^{\prime \prime}\end{array}$ & OFSECV & 43.1 & & & & & 1 & & 1 \\
\hline & Secundus ii १०f & OFSECVN & 3.1 & & & & 2 & & & 2 \\
\hline lucundus iii & SEX.IVL.IVCVND & $\begin{array}{l}\text { SEX.IVL. } \\
\text { IVCVND }\end{array}$ & 2.9 & & & & & 50 & & 50 \\
\hline Silvius i & Silvius i 9a & OFSILV & 16.1 & & 5 & & & & & 5 \\
\hline$(\mathrm{T}())(1))$ & $(\mathrm{T}() \|())$ & $\mathrm{T}() \|()$ & 30.1 & & & & 1 & & & 1 \\
\hline (TABIVIMSS) & (TABIVIMSS) & TABIVIMSS & 14.1 & 13 & 136 & & & & & 149 \\
\hline (VA?ON) & (VA?ON) & VA?ON & 22.1 & & & & 2 & & & 2 \\
\hline (VEEI?) & (VEEI?) & VEEI & 17.1 & & 10 & & & & & 10 \\
\hline (Virthus) & (Virthus) & VIRTHV & 45.1 & & & & & 192 & & 192 \\
\hline (Virtus) & (Virtus) & VIRTVTIS & 6.1 & & & & 7 & & & 7 \\
\hline (Vitalis) & (Vitalis) & OFVITA & 37.1 & & & & & 4 & & 4 \\
\hline
\end{tabular}




\begin{tabular}{|c|c|c|c|c|c|c|c|c|c|c|}
\hline Potter & Die number & Transcription & $\begin{array}{r}\text { Reference to } \\
\text { Nieto et al. } \\
1989\end{array}$ & $\begin{array}{l}\text { Drag. } \\
24 / 25\end{array}$ & Drag. 27 & $\begin{array}{l}\text { Drag. } \\
15 / 17\end{array}$ & Drag. 18 & Drag. 29 & $\begin{array}{r}\text { Hermet } \\
1\end{array}$ & Total \\
\hline \multirow[t]{9}{*}{ illiterate } & illiterate & illiterate & 46.1 & & & & & 1 & & 1 \\
\hline & illiterate & illiterate & 44.1 & & & & & 1 & & 1 \\
\hline & illiterate & illiterate & 18.1 & & & & 2 & & & 2 \\
\hline & illiterate & illiterate & 19.1 & & & & 3 & & & 3 \\
\hline & illiterate & illiterate & 23.1 & & & & 2 & & & 2 \\
\hline & illiterate & illiterate & 32.1 & & & & 1 & & & 1 \\
\hline & illiterate & illiterate & 26.1 & 4 & 1 & & & & & 5 \\
\hline & illiterate & illiterate & 27.1 & & 2 & & & & & 2 \\
\hline & illiterate & illiterate & 33.1 & & 2 & & & & & 2 \\
\hline \multirow[t]{2}{*}{ unidentified } & unidentified & unidentified & 29.1 & & & & 2 & 2 & & 4 \\
\hline & & & & 232 & 439 & 85 & 584 & 345 & 2 & 1687 \\
\hline
\end{tabular}


T A B L E 3. C O L C H E T E R F I R T S H O P

\begin{tabular}{|c|c|c|c|c|c|c|c|}
\hline Potter & Die number & Drag. 24/25 & Drag. 27 & Drag. 15/17 & Drag. 18 & $\begin{array}{r}\text { Drag. } 15 / 17 \\
\text { or } 18 \\
\end{array}$ & Total \\
\hline Abitus & Abitus 1a & 1 & & & & & 1 \\
\hline Aquitanus & Aquitanus 21a & & 1 & & & & 1 \\
\hline Bassus i & Bassus i 15a & 3 & & & & & 3 \\
\hline Bassus i-Coelus & Bassus i - Coelus 6b & 1 & & & & & 1 \\
\hline Bio & Bio 10a & & 1 & & & & 1 \\
\hline Chrestus & Chrestus 3a & & & & 1 & 1 & 2 \\
\hline Icdo & Icdo 1a & & 3 & & & & 3 \\
\hline \multirow[t]{2}{*}{ Masc(u)lus i } & Masc(u)lus i 5b & & 1 & & & & 1 \\
\hline & Masc(u)lus i 7a & 1 & & & & & 1 \\
\hline Maso i & Maso i 1a & & & & & 2 & 2 \\
\hline Modestus i & Modestus i 2e & & & & 1 & & 1 \\
\hline Murranus & Murranus 10c & & & & & 2 & 2 \\
\hline Nestor & Nestor 2a & & & & & 1 & 1 \\
\hline Pass(i)enus & Pass(i)enus 50a & & & & & 1 & 1 \\
\hline Paullus i & Paullus i 9a & & 1 & & & & 1 \\
\hline \multirow[t]{5}{*}{ Primus iii } & Primus iii 12c & & & 1 & 7 & 4 & 12 \\
\hline & Primus iii $12 q$ & 1 & 2 & & & & 3 \\
\hline & Primus iii 18b & 3 & & & & & 3 \\
\hline & Primus iii 20b & & & & & 1 & 1 \\
\hline & Primus iii 21i & 4 & 3 & & & & 7 \\
\hline Tertius ii & Tertius ii 15a & 3 & & & & & 3 \\
\hline \multirow[t]{2}{*}{ Virtus i } & Virtus i 11a & 11 & & & & & 11 \\
\hline & & 28 & 12 & 1 & 9 & 12 & 62 \\
\hline
\end{tabular}


T A B L E 4. C O L C H E T ER S E C O N D S H O P

\begin{tabular}{|c|c|c|c|c|c|c|c|c|c|}
\hline Potter & Die number & Drag. $24 / 25$ & Drag. 27 & Drag. 15/17 & Drag. 18 & $\begin{array}{r}\text { Drag. } 15 / 17 \\
\text { or } 18\end{array}$ & Drag. 29 & Ritt. 8 & Total \\
\hline Abitus & Abitus $9 \mathrm{~b}$ & & & & 1 & & & & 1 \\
\hline Albinus i & Albinus i 3a & & & & & 1 & & & 1 \\
\hline Aquitanus & Aquitanus 6a & & 14 & & & & & & 14 \\
\hline Bio & Bio 2b & 1 & & & & & & & 1 \\
\hline Celer ii & Celer ii 8a & & & & 1 & & & & 1 \\
\hline Chrestus & Chrestus 3a' & & & & 1 & & & & 1 \\
\hline \multirow[t]{2}{*}{ Felix i } & Felix i 2d & & & & & & 4 & & 4 \\
\hline & Felix i 6b & & 5 & & & & & & 5 \\
\hline lustus i & lustus i 7a & & & & & & & 2 & 2 \\
\hline \multirow[t]{2}{*}{ Licinius } & Licinius 7a & & & & 1 & & & & 1 \\
\hline & Licinius 23a & & 5 & & & & & & 5 \\
\hline Magnus i & Magnus i 1a & & & & 1 & & & & 1 \\
\hline Marinus & Marinus i 4a & & & & & & 1 & & 1 \\
\hline Max... i & Max... i 1a & 4 & & & & & & & 4 \\
\hline Modestus i & Modestus i 4b' & & 1 & & & & & & 1 \\
\hline Mommo & Mommo 26d & & 9 & & & & & & 9 \\
\hline Montanus & Montanus i 4a & & & & 3 & & & & 3 \\
\hline Nestor & Nestor 2a & & & & 3 & & & & 3 \\
\hline Pass(i)enus & Pass(i)enus 50a & & & & & 1 & & & 1 \\
\hline \multirow[t]{6}{*}{ Primus iii } & Primus iii 12c & & & & 2 & & & & 2 \\
\hline & Primus iii 12r & & & & 1 & & & & 1 \\
\hline & Primus iii 12v & & & & 1 & & & & 1 \\
\hline & Primus iii 18b & 1 & & & & & & & 1 \\
\hline & Primus iii 20b & & 1 & & & & & & 1 \\
\hline & Primus iii 27a & & & & 1 & & & & 1 \\
\hline Primus iii-Sco & Primus iii-Sco... 6a & & & 1 & & & & & 1 \\
\hline Scotnus & Scotnus 5a & & & & 1 & & & & 1 \\
\hline Virtus & Virtus i 11a & 1 & & & & & & & 1 \\
\hline .MYY & .MYY (illiterate) & & 2 & & & & & & 2 \\
\hline \multirow[t]{3}{*}{ unknown } & ()N (incomplete) & & & & 1 & & & & 1 \\
\hline & O() (incomplete) & & & & 1 & & & & 1 \\
\hline & & 7 & 37 & 1 & 19 & 2 & 5 & 2 & 73 \\
\hline
\end{tabular}


